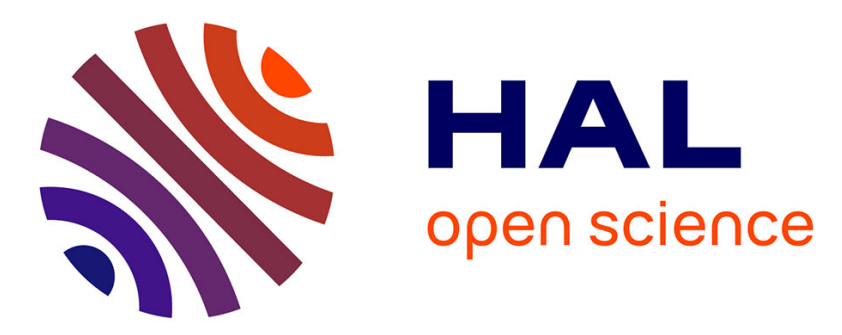

\title{
A direct method for predicting the high-cycle fatigue regime of shape-memory alloys structures
}

\author{
Michaël Peigney
}

\section{To cite this version:}

Michaël Peigney. A direct method for predicting the high-cycle fatigue regime of shape-memory alloys structures. Advances in Direct Methods for Materials and Structures, Springer, pp.13-28, 2018, 9783-319-59810-9. 10.1007/978-3-319-59810-9_2 . hal-01781452

\section{HAL Id: hal-01781452 \\ https://hal-enpc.archives-ouvertes.fr/hal-01781452}

Submitted on 30 Apr 2018

HAL is a multi-disciplinary open access archive for the deposit and dissemination of scientific research documents, whether they are published or not. The documents may come from teaching and research institutions in France or abroad, or from public or private research centers.
L'archive ouverte pluridisciplinaire HAL, est destinée au dépôt et à la diffusion de documents scientifiques de niveau recherche, publiés ou non, émanant des établissements d'enseignement et de recherche français ou étrangers, des laboratoires publics ou privés. 


\title{
A direct method for predicting the high-cycle fatigue regime of shape-memory alloys structures
}

\author{
Michaël Peigney
}

\begin{abstract}
Shape Memory Alloys (SMAs) belong to the class of so-called smart materials that offer promising perspectives in various fields such as aeronautics, robotics, biomedicals or civil engineering. For elastic-plastic materials, there is an established correlation between fatigue and energy dissipation. In particular, highcycle fatigue occurs when the energy dissipation remains bounded in time. Although the physical mechanisms in SMAs differ from plasticity, the hysteresis that is commonly observed in the stress-strain response of those materials shows that some energy dissipation occurs. It can be reasonably assumed that situations where the energy dissipation remains bounded are the most favorable for fatigue durability. In this contribution, we present a direct method for determining if the energy dissipation in a SMA structure (submitted to a prescribed loading history) is bounded or not. That method is direct in the sense that nonlinear incremental analysis is completely bypassed. The proposed method rests on a suitable extension of the wellknown Melan theorem. An application related to biomedical stents is presented to illustrate the method.
\end{abstract}

\section{Introduction}

The peculiar properties of Shape Memory Alloys (SMAs) - such as the superelastic behavior or the shape memory effect - are the result of a solid/solid phase transformation between different crystallographic structures (known as austenite and martensite). That phase transformation takes place at the microscopic level and is driven both by thermal and mechanical loading. The crystallographic structure of the austenite is more symmetric than the crystallographic structure of the martensite. This leads one to distinguish between several symmetry related martensitic variants

Michaël Peigney

Université Paris-Est, Laboratoire Navier (UMR 8205), CNRS, Ecole des Ponts ParisTech, IFST-

TAR, F-77455 Marne la Vallée, France, e-mail: michael.peigney@ polytechnique.org 
corresponding to different orientations of the martensitic lattice with respect to the austenitic lattice [7]. Each martensitic variant is characterized by a transformation strain that describes the deformation from the austenitic lattice to the martensitic lattice.

Shape memory alloys can be relevant in a lot of applications, but for the time being the most successful applications are to be found in the biomedical domain, for devices such as endovascular stents. In vivo, such devices are submitted to a cyclic loading due to the cardiac cycle, and therefore may be subjected to fatigue [ 9 , $28,30]$. Metal fatigue is a crucial issue for structures submitted to variable loading. A conventional way to estimate the fatigue behavior of a material is to establish the so-called S-N curves that show the number of cycles to failure $\mathrm{N}$ versus the loading amplitude. Three different regimes are typically observed on S-N curves:

- the low-cycle fatigue regime, corresponding to $\mathrm{N}<10^{4}-10^{5}$ cycles. In that regime, $\mathrm{N}$ decreases rapidly with the loading amplitude $\mathrm{S}$.

- the high-cycle fatigue regime, corresponding to $\mathrm{N}>10^{4}-10^{5}$ cycles, for which the decrease of $\mathrm{N}$ with the loading amplitude $\mathrm{S}$ is much slower.

- the unlimited lifetime regime, in which the material shows no sign of fatigue. That behaviour is observed for loading amplitudes $\mathrm{S}$ smaller than a characteristic value referred to as the endurance limit.

The demarcation between low- and high-cycle fatigue depends on the material considered [17]. Similarly, depending on the material considered, the endurance limit may exist or not. For Nitinol (which is the most common shape memory alloy used in applications), the three regimes listed above have been observed in cyclic traction experiments [28].

For designing Shape Memory Alloys structures subjected to variable loading, it is essential to have tools for assessing the fatigue life. A case in point is the design of biomedical Nitinol stents. Since biomedical stents are required to have high durability, it is essential to make sure that those devices operate in the high-cycle fatigue regime or -even better- in the unlimited lifetime regime. Although they provide some valuable insight in the fatigue behavior, $\mathrm{S}-\mathrm{N}$ curves are uniaxial in nature and therefore are not sufficient for estimating the fatigue behavior of complex threedimensional structures subjected to multiaxial loading.

This contribution presents a rational method for predicting the high-cycle fatigue regime of SMA structures. That method is based on the principle that high-cycle fatigue corresponds to situations where phase transformation is limited, i.e. the energy dissipation is bounded. By analogy with plasticity, that situation is referred to as shakedown. The proposed method relies on recent theoretical results that give a sufficient condition for shakedown to occur in SMA structures submitted to variable loading.

This contribution is organized as follows: We start by setting some notations and making some observations on the constitutive laws commonly used for SMAs. From there we comment on the structural evolution problem, with a special emphasis on the large time behavior and recent results regarding the shakedown behavior. This leads us to propose a direct method for predicting the high-cycle fatigue regime. 
The implementation of that method is detailed for a parametrized loading history. An application related to biomedical stents is presented to illustrate the method. In particular, the results delivered by the proposed method are compared with experimental results from the literature.

\section{Constitutive laws}

In most existing material models for SMAs, the strain $\varepsilon$ is decomposed in an elastic part $M: \sigma$ proportional to the stress $\sigma$ and an inelastic part $K: \alpha$ related to phase transformation, i.e.

$$
\varepsilon=M: \sigma+K: \alpha .
$$

In Eq.(1), $M$ is the elasticity tensor, $K$ is a fixed tensor, and $\alpha$ is an internal variable that tracks the phase transformation. The Helmholtz energy $w$ corresponding to (1) is of the form

$$
w(\varepsilon, \alpha)=\frac{1}{2}(\varepsilon-K: \alpha): M^{-1}:(\varepsilon-K: \alpha)+f(\alpha)
$$

where $f$ is a positive function of $\alpha$ whose expression depends on the model considered (some examples will be given later on). In the following, we denote by $A$ the thermodynamical force associated with $\alpha$, as defined by

$$
A=-\frac{\partial w}{\partial \alpha}=K^{T}: \sigma-f^{\prime}(\alpha)
$$

where $K^{T}$ is the transpose of $K$.

To account for hysteresis effects, Eq.(1) is complemented with an elasticity domain $\mathscr{C}$ and a flow rule (describing the evolution of $\alpha$ ) akin to plasticity. The elasticity domain is assumed to be convex and to contain the origin. The normality flow rule is commonly used, i.e.

$$
\dot{\alpha} \in \partial \mathrm{I}_{\mathscr{C}}(A)
$$

where the dot ${ }^{\circ}$ denotes left-time differentiation and $\partial \operatorname{I}_{\mathscr{C}}(A)$ is the normal cone of the elasticity domain $\mathscr{T}$ at point $A$, defined by

$$
\partial_{\mathscr{C}}(A)=\left\{g \mid g: A \geq g: A^{\prime} \text { for any } A^{\prime} \in \mathscr{C}\right\} .
$$

For later reference, we note that the normality flow rule (4) respects the principle of maximum dissipation

$$
\dot{\alpha}:\left(A-A^{\prime}\right) \geq 0 \forall A^{\prime} \in \mathscr{C} .
$$

As a first example, consider the model of Souza et al.[33]. In that model, the internal variable $\alpha$ is a deviatoric strain (referred to as the transformation strain) and $K$ is taken as the projector on the deviatoric space, i.e. 


$$
K=\mathbb{I}-\frac{1}{3} \mathbb{1} \otimes \mathbb{1}
$$

where $\mathbb{1}$ and $\mathbb{I}$ are respectively the second- and fourth-order identity tensors. In such a model, the thermodynamical force $A$ specializes as

$$
A=s-f^{\prime}(\alpha)
$$

where $s$ is the deviatoric stress. The elasticity domain $\mathscr{C}$ considered by Souza et al. is of the Von Mises type, i.e. defined by $\left\|s-f^{\prime}(\alpha)\right\| \leq R$ where $\|\cdot\|$ is the Euclidean norm and $R$ is the yield limit for phase transformation. In addition, the internal variable $\alpha$ is submitted to the constraint $\|\alpha\| \leq \varepsilon_{L}$, which expresses the fact that the transformation strain cannot be arbitrarily large and is bounded by some material parameter $\varepsilon_{L}$ that depends on the alloy considered.

A typical superelastic stress-strain delivered by such a model is represented in Fig. 1. Note in particular that $\varepsilon_{L}$ is the strain amplitude of the plateaux exhibited by the stress-strain response. The hysteresis displayed by the stress-strain curve is directly related to the energy dissipated in a strain-driven loading cycle of sufficiently large amplitude $\Delta \varepsilon$ as represented in Fig. 1. If such a cyclic strain is applied to the material, energy dissipation would occur at each cycle. In such a condition, the material would be subjected to low-cycle fatigue. In contrast, if a cyclic strain of low amplitude $\Delta \varepsilon$ is imposed (possibly around a non-zero mean value), then there would be no dissipation in the stabilized regime so that high-cycle fatigue will prevail (Fig. 2).

The model of Souza et al., as well as its further refinements and extensions [4, 5] are phenomenological. In contrast, other SMA models rely on a micromechanical approach and make use of detailed information on the crystallography of the phase transformation $[2,12,13,24,25]$. The simplest case is that of single crystals: the internal variable is typically chosen as $\left(\theta_{1}, \theta_{k}\right)$ where $\theta_{i}$ is the volume fraction of the martensite variant $i$. The stress-strain relation (1) specializes as

$$
\varepsilon=M: \sigma+\sum_{i=1}^{k} \theta_{i} \varepsilon_{i}^{t r, 0}
$$

Fig. 1 Superelastic stressstrain response for a loading cycle of large strain amplitude $\Delta \varepsilon$.

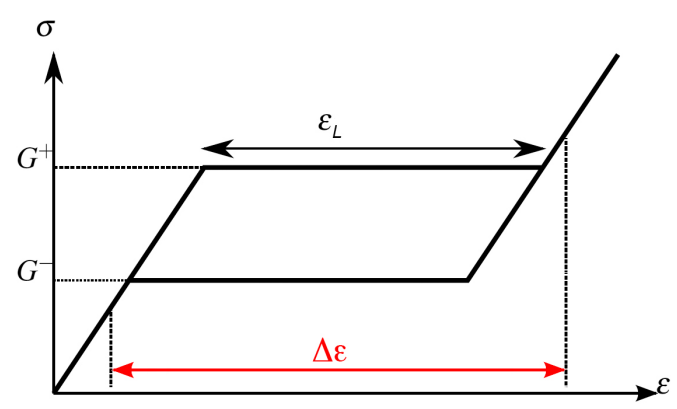


where $\varepsilon_{i}^{t r, 0}$ is the transformation strain for variant $i$ and $k$ is the number of martensitic variants [7]. Both $k$ and $\varepsilon_{i}^{t r, 0}(i=1, \cdots, k)$ are to be considered as material parameters. As mentioned in Sect. 1 , the transformation strains $\varepsilon_{i}^{t r, 0}$ are symmetry-related i.e. for any $(i, j)$ there exists a rotation $R_{i j}$ such that

$$
\varepsilon_{i}^{t r, 0}=R_{i j}^{T} \cdot \varepsilon_{j}^{t r, 0} \cdot R_{i j}
$$

A common choice is to define the elasticity domain by

$$
\max _{i}\left|\varepsilon_{i}^{t r, 0}: \sigma-f_{, i}\right| \leq G
$$

where $G$ is a yield limit for phase transformation and $f_{, i}$ is the partial derivative of $f\left(\theta_{1}, \cdot, \theta_{n}\right)$ with respect to $\theta_{i}$. Note that the internal variable $\left(\theta_{1},, \theta_{k}\right)$ is bounded because the volume fractions $\theta_{i}$ are positive and their sum is less than 1 .

Such a micromechanical approach can be extended to polycrystals, which is the common form of commercially produced SMAs. A polycrystal is an assemblage of $N$ crystalline orientations. Each orientation $j$ is characterized by a rotation $R_{j}$ with respect to a reference orientation (which can be chosen to coincide with that of the single crystal considered in (7)). In most of micromechanical models of polycrystalline SMAs $[13,19,20]$, the internal variable is taken as $\alpha=\left(\theta_{11}, \cdot, \theta_{k N}\right)$ where $\theta_{i j}$ is the volume fraction of martensite variant $i$ in the crystalline orientation $j$. The internal variable $\alpha=\left(\theta_{11}, \theta_{k N}\right)$ is submitted to the constraint

$$
0 \leq \theta_{i j}, \sum_{i=1}^{k} \theta_{i j}=c_{j}
$$

where $c_{j}$ is the volume fraction of orientation $j$. The stress-strain relation and the elasticity domain are respectively defined by

$$
\varepsilon=M: \sigma+\sum_{i=1}^{k} \sum_{j=1}^{N} \theta_{i} \varepsilon_{i j}^{t r}
$$

and

Fig. 2 Superelastic stressstrain response for a loading cycle of small strain amplitude $\Delta \varepsilon$.

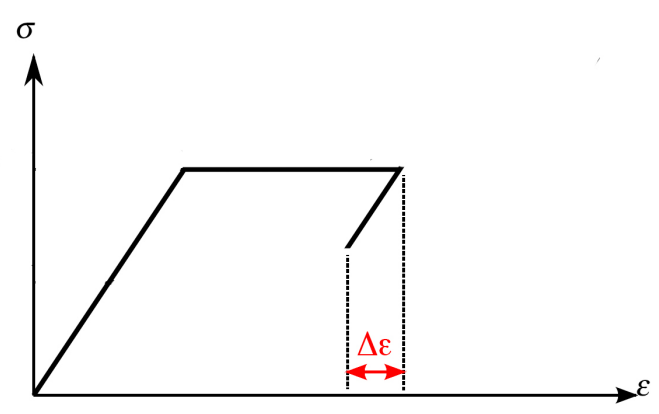




$$
\max _{i, j}\left|\varepsilon_{i j}^{t r}: \sigma-f_{, i j}\right| \leq G
$$

where $f_{, i j}$ is the partial derivative of $f$ with respect to $\theta_{i j}$ and $\varepsilon_{i j}^{t r}$ is the transformation strain of variant $i$ in the crystalline orientation $j$. The transformation strain $\varepsilon_{i j}^{t r}$ can be written as

$$
\varepsilon_{i j}^{t r}=R_{j}^{T} \cdot \varepsilon_{i}^{t r, 0} \cdot R_{j} .
$$

In all the models mentioned, observe that the internal variable $\alpha$ used for tracking the phase transformation is always bounded. This requirement can be written as

$$
\alpha \in \mathscr{T}
$$

where $\mathscr{T}$ is the bounded set of admissible values for $\alpha$. For instance, in the model of Souza et al., the set $\mathscr{T}$ is the set of deviatoric strains with norm less than $\varepsilon_{L}$. The constraint (10) is a distinctive feature of SMAs (compared to plasticity) and stems from the mass conservation in the phase transformation process. Rigorously speaking, in the presence of such constraints, the normality flow rule (4) needs to be modified as

$$
\dot{\alpha} \in \partial I_{\mathscr{C}}\left(A-A^{r}\right) ; A^{r} \in \partial I_{\mathscr{T}}(\alpha)
$$

where $\partial I_{\mathscr{T}}(\alpha)$ is the normal cone of $\mathscr{T}$ at point $\alpha$ and is defined in a similar fashion as in Eq.(5). For a convex set $\mathscr{T}$ (which is assumed throughout this chapter), the following inequality - formally similar to (6) - holds [8]:

$$
A_{r}:\left(\alpha-\alpha^{\prime}\right) \geq 0 \forall \alpha^{\prime} \in \mathscr{T} .
$$

Reference is made to [11] for a derivation of (11) from the general principles of thermodynamics. The term $A_{r}$ in (12) can be interpreted as a 'reaction force' and is non zero only when $\alpha$ saturates the constraint (i.e. when $\alpha$ is on the boundary of $\mathscr{T})$.

\section{Structural evolution problem}

Now consider a structure occupying a domain $\Omega$ and submitted to a given loading history. For determining the evolution of the structure, the constitutive laws (1-1011 ) are to be satisfied at each point $x$ and at each time $t$. In addition, the stress field needs to satisfy the equilibrium equations and the strain field has to derive from a displacement field that respects the boundary conditions, i.e.

$$
\sigma \in \mathscr{A}_{\sigma}(t), \varepsilon \in \mathscr{A}_{\varepsilon}(t)
$$

where $\mathscr{A}_{\sigma}(t)$ and $\mathscr{A}_{\varepsilon}(t)$ are respectively the sets of statically admissible stress and kinematically admissible strain fields at time $t$, defined by 


$$
\begin{aligned}
& \mathscr{A}_{\sigma}(t)=\left\{\sigma \mid \operatorname{div} \sigma+f^{d}=0 \text { in } \Omega ; \sigma \cdot n=T^{d} \text { on } \Gamma_{T}\right\}, \\
& \mathscr{A}_{\varepsilon}(t)=\left\{\varepsilon \mid \varepsilon=\left(\nabla u+\nabla^{T} u\right) / 2 \text { in } \Omega ; u=u^{d} \text { on } \Gamma_{u}\right\} .
\end{aligned}
$$

In (14), $f^{d}, T^{d}$ and $u^{d}$ are functions of $(x, t)$ and define the loading history. The tractions $T^{d}$ and the displacements $u^{d}$ are applied respectively on a part $\Gamma_{T}$ and a part $\Gamma_{u}=\partial \Omega-\Gamma_{T}$ of the boundary $\partial \Omega$.

Together the equations (1-10-11-13) define the structural evolution problem. That problem is nonlinear and is usually solved incrementally using space- and timediscretization techniques, resorting for instance to Finite Element Analysis (FEA). Regarding numerical implementation, handling the constraint (10) on the internal variable is an additional difficulty compared to plasticity [3, 4, 31], especially for micromechanical models [12, 22, 23].

Asides from numerical issues, adding a constraint (10) has a profound impact on the properties of the structural evolution problem, especially regarding the largetime behavior. For large loadings, the large-time behavior is indeed strongly depen-

dent on the initial state. For instance, in the case of periodic loading, some initial conditions may lead to shakedown while others may lead to alternate phase transformation (i.e. a periodic but non constant evolution of the phase transformation) $[21,26]$. Interestingly, such dependence of the asymptotic regime on the initial state has also been observed in other nonlinear mechanical problems, such as contact with friction [1] and plasticity with temperature-dependent elastic moduli [27].

\section{Shakedown theorem}

For fatigue design, we are especially interested in situations where the solutions of the structural evolution problem are such that the energy dissipation remains bounded in time. That situation is referred to as shakedown and corresponds to the most favorable case of high-cycle fatigue. In standard plasticity, the Melan's theorem is a well-known result that gives a sufficient condition for shakedown to occur $[18,34,15]$. Melan's theorem is path-independent, i.e. the obtained shakedown condition is independent of the initial state. The original theorem cannot be directly applied to SMAs because, contrary to standard plasticity, the internal variable is bounded. The theoretical issue of extending Melan theorem to SMAs has been addressed in $[10,21,26]$. In particular, a path-independent Melan's theorem has been obtained in $[21,26]$.

Let $\left(\sigma^{E}, \varepsilon^{E}\right)$ be the fictitious elastic response of the structure, defined by the elasticity problem

$$
\varepsilon^{E}=M: \sigma^{E}, \sigma^{E} \in \mathscr{A}_{\sigma}(t), \varepsilon^{E} \in \mathscr{A}_{\varepsilon}(t)
$$

The statement of the theorem is the following:

Theorem 1. If there exists $m>1, \tau \geq 0$ and a time-independent field $A_{*}^{r}(x)$ such that 


$$
m K^{T}: \sigma^{E}(x, t)-A_{*}^{r}(x) \in \mathscr{C} \forall x \in \Omega, \forall t>\tau
$$

then there is shakedown, whatever the initial condition is.

The proof of Theorem 1 is given in the Appendix. Some comments are in order. A first observation is that Theorem 1 is path-independent, just as the standard Melan theorem in perfect plasticity: If the condition in Theorem 1 is satisfied, then shakedown occurs for all initial state. The shakedown condition provided by Theorem 1 is thus independent of any residual stress that may exist initially in the structure (as a consequence of material process, for instance). When the loading is beyond the limit provided by Theorem 1, shakedown may still occur for some (but not all) initial conditions (see [21, 26] for some examples). In such case, the asymptotic behavior is strongly dependent on the initial state.

Observe also that the field $A_{*}^{r}(x)$ in Theorem 1 is free from any constraint. This makes for a simple geometrical interpretation of Theorem 1: Shakedown occurs if, up to a time-independent translation, the local elastic response $t \mapsto K^{T}: \sigma^{E}(x, t)$ remains in the elasticity domain $\mathscr{C}$ at each point $x$ (Fig. 3 ). The situation is reminiscent of linear kinematic hardening plasticity, for which shakedown is ensured under a similar condition [16].

On a final note, we observe that Theorem 1 is largely independent of the details of the SMA model considered. In particular, the function $f$ (that appears in the Helmholtz energy $w$ in Eq. (2) ) and the exact expression of the set $\mathscr{T}$ (that defines the constraints on the internal variable in Eq. (10)) do not play a role in Theorem 1.

\section{Description of the method}

The above theorem leads to a design method against fatigue that can be broken down into two steps:

1. Calculate the elastic response $\sigma^{E}(x, t)$ for the considered loading history.

2. Check if the local curve $t \mapsto \sigma^{E}(x, t)$ can be translated in $\mathscr{C}$ at each point $x$.

Note that Step 1. can be conveniently performed by a (linear elastic) FEA. Step 2. is merely a post-processing of the results obtained in Step 1. Consider for instance a

Fig. 3 Geometric interpretation of the local shakedown condition for shape memory alloys.

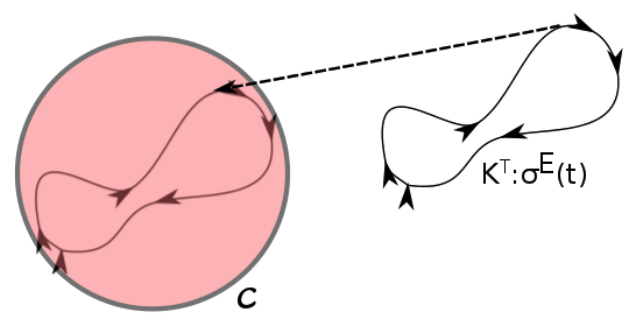


parametrized loading history: The loading consists of body forces $f^{d}(x, t)$, applied tractions $T^{d}(x, t)$ and prescribed displacements $u^{d}(x, t)$ that vary respectively as

$$
f^{d}(x, t)=\lambda(t) f_{0}(x), T^{d}(x, t)=\lambda(t) T_{0}(x), u^{d}(x, t)=\lambda(t) u_{0}(x)
$$

where $\lambda(t)$ is a time-dependent loading parameter. The elastic response $\sigma^{E}(x, t)$ being defined by a linear problem, we have

$$
\sigma^{E}(x, t)=\lambda(t) \sigma_{0}^{E}(x)
$$

where $\sigma_{0}^{E}(x)$ is the elastic stress field for the loading $\left(f_{0}(x), T_{0}(x), u_{0}(x)\right)$. At each point $x$, the curve $t \mapsto K^{T}: \sigma^{E}(x, t)$ thus describes the line segment

$$
\left[\lambda_{\min }, \lambda_{\max }\right] K^{T}: \sigma_{0}^{E}(x)
$$

where $\lambda_{\min }=\min _{t} \lambda(t)$ and $\lambda_{\max }=\max _{t} \lambda(t)$.

Details of Step 2. depends on the model used. The simplest case is that of phenomenological models, such as the model of Souza et al.[33] considered previously. In that model, the elasticity domain $\mathscr{C}$ is a ball of radius $R$ in the deviatoric space. Denoting by $s_{0}^{E}(x)$ the deviatoric part of $\sigma_{0}^{E}$, performing Step 2. amounts to checking that

$$
\left(\lambda_{\max }-\lambda_{\min }\right)\left\|s_{0}^{E}(x)\right\|<2 R
$$

at each point $x$, which is guaranteed if

$$
\left(\lambda_{\max }-\lambda_{\min }\right) \max _{x}\left\|s_{0}^{E}(x)\right\|<2 R .
$$

If that condition is satisfied, then the structure experiences high-cycle fatigue, whatever the initial state is. In practice, the condition (17) can be used as a criterion for the design of SMA structures against fatigue: For say a given loading history, the geometry of the structure should be designed in such fashion that $\max _{x}\left\|s_{0}^{E}(x)\right\|$ remains smaller than $2 R /\left(\lambda_{\max }-\lambda_{\min }\right)$. Note that the exact knowledge of the loading parameter $\lambda(t)$ is not necessary: Only bounds on the extreme values are needed. In particular, $\lambda(t)$ does not need to be periodic in time.

Now consider a micromechanical model of polycystalline SMAs. Performing Step 2. amounts to check whether there exists $A_{i j}^{*}(x)$ and $m>1$ such that

$$
\left|m \lambda(t) \sigma_{0}^{E}(x): \varepsilon_{i j}^{t r}-A_{i j}^{*}(x)\right| \leq G \forall(i, j)
$$

i.e. that

$$
\left(\lambda_{\max }-\lambda_{\min }\right)\left|\sigma_{0}^{E}(x): \varepsilon_{i j}^{t r}\right|<2 G \forall(i, j) .
$$

This last requirement can be rewritten as

$$
\left(\lambda_{\max }-\lambda_{\min }\right) \max _{i, j}\left|\sigma_{0}^{E}(x): \varepsilon_{i j}^{t r}(x)\right|<2 G .
$$


Proceeding further requires to evaluate $\max _{i, j}\left|\sigma_{0}^{E}(x): \varepsilon_{i j}^{t r}(x)\right|$. This can be done exactly provided that the polycrystalline texture (i.e. the list of $N$ rotations that appear in (9) ) is given. As an alternative, a simple bound on $\max _{i, j}\left|\sigma_{0}^{E}(x): \varepsilon_{i j}^{t r}\right|$ can be used, as is now explained. For any $(i, j)$ we have indeed

$$
\sigma_{0}^{E}(x): \varepsilon_{i j}^{t r}=\frac{1}{3} \operatorname{tr} \sigma_{0}^{E}(x) \operatorname{tr} \varepsilon_{i j}^{t r}+s_{0}^{E}(x): \varepsilon_{i j}^{t r, D} \leq \frac{1}{3} \operatorname{tr} \sigma_{0}^{E}(x) \operatorname{tr} \varepsilon_{i j}^{t r}+\left\|s_{0}^{E}(x)\right\| \cdot\left\|\varepsilon_{i j}^{t r, D}\right\|
$$

where the superscript ${ }^{D}$ denotes the deviatoric part. Two observations are in order. The first one is that, for the most common shape memory alloys, the transformation strains can be considered as trace-free [7]. The second observation is that, as a result of (7-9), $\left\|\varepsilon_{i j}^{t r, D}\right\|$ takes a constant value (denoted by $\left\|\varepsilon^{t r}\right\|$ ), independently of $(i, j)$. It follows that $\sigma_{0}^{E}(x): \varepsilon_{i j}^{t r} \leq\left\|\varepsilon^{t r}\right\| \cdot\left\|s_{0}^{E}(x)\right\|$ for all $(i, j)$, hence

$$
\max _{i, j} \sigma_{0}^{E}(x): \varepsilon_{i j}^{t r} \leq\left\|\varepsilon^{t r}\right\| \cdot\left\|s_{0}^{E}(x)\right\| .
$$

In view of (18) and (19), a sufficient condition for shakedown to occur is thus that

$$
\left(\lambda_{\max }-\lambda_{\min }\right)\left\|\varepsilon^{t r}\right\| \cdot\left\|s_{0}^{E}(x)\right\|<2 G
$$

for all $x \in \Omega$. That condition is satisfied if

$$
\left(\lambda_{\max }-\lambda_{\min }\right) \max _{x}\left\|s_{0}^{E}(x)\right\|<2 \frac{G}{\left\|\varepsilon^{t r}\right\|}
$$

Observe that the final condition (20) is formally similar to that obtained in (17) for a phenomenological model.

\section{Application to biomedical stents}

We now describe the application of the proposed method to biomedical stents. Such devices have a tubular geometry and are typically an assemblage of elementary cells. Those cells often have the shape of a 'strut V', as represented in Fig. 4 (left). When the stent is loaded radially (for instance a consequence of blood pressure), each cell primarily experiences some uniaxial traction (along the horizontal direction in Fig. 4 ). Extensive fatigue tests have been reported in [2]. Those tests were performed on a diamond-shape specimen that consists of 2 'strut Vs' arranged in a symmetric fashion so as to be easily fitted in a fatigue test machine. A simplified model of such specimen is shown in Fig. 4 (right). In the experiments reported in [2], each sample was submitted to a given strain cyclically between a fixed minimum value $\varepsilon_{\min }$ and a maximum value $\varepsilon_{\max }$. The number of cycles to failure was recorded for each sample. The obtained experimental results showed that a low- to high-cycle fatigue transition occurs at $0.4-0.5 \%$ strain amplitude, without any clear influence of the mean strain. In order to illustrate the proposed approach based on shakedown 
theory, we apply the method detailed previously and compare the predictions with the experimental results in [2].

For the problem at hand, applying the proposed approach merely consists in performing one single elastic calculation, namely calculating the elastic response of the structure in Fig. 4 when it is submitted to a (arbitrary fixed) reference strain $\varepsilon_{0}$. Such a calculation has been performed in 2D (plane stress) with the FEA software Freefem [14]. We used the values $E=50 \mathrm{GPa}, v=0.35$ which are representative of Nitinol [33]. Because of the symmetries, only one fourth of the structure needs to be modeled, as represented in Fig. 4. The mesh used in the FEA consists of 121847 triangular elements with linear interpolation. In Fig. 5 is represented the map of $\left\|s_{0}^{E}(x)\right\|$. The maximum value of $\left\|s_{0}^{E}(x)\right\|$ (normalized with respect to $\varepsilon_{0}$ ) is approximatively equal to $6860 \mathrm{MPa}$.

Using the condition (17) with $R=40 \mathrm{MPa}$, we obtain that shakedown occurs provided that

$$
\left|\varepsilon_{\max }-\varepsilon_{\min }\right|<0.58 \% .
$$

Using lattice parameters from the literature [7], the value $\left\|\varepsilon^{t r}\right\|$ for Nitinol is found to be approximatively equal to 0.1048 . Applying the conditions (20) with $G=4.7$ $\mathrm{MPa}$ [2], we obtain that shakedown occurs for

$$
\left|\varepsilon_{\max }-\varepsilon_{\min }\right|<0.65 \% .
$$

Both values in (21) and (22) are above the low- to fatigue transition that is assessed experimentally (0.4-0.5\%). However, given the uncertainties in the material parameters and the lack of data on the exact geometry used in the experiments, the agreement with the experimental results can be considered as satisfactory. Also note that the predicted limit is independent of the mean applied strain, which is in line with the experiments.

Fig. 4 Strut V (left) and diamond-shaped specimen

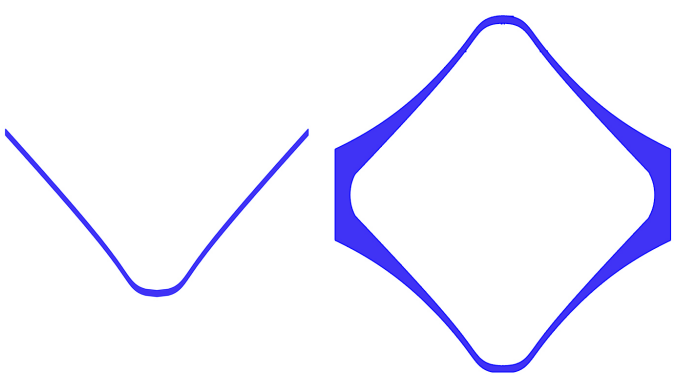
(right) 


\section{Conclusions}

The proposed method is relevant for the fatigue design of SMA structures. It enjoys the following key features:

1. It is based on rational theoretical results.

2. It can be used with a lot of existing SMA models (either phenomenological or micromechanical).

3. It only relies on elastic calculations: Incremental nonlinear analysis is bypassed.

4. Only a partial knowledge of the loading is required (namely the knowledge of the extreme values).

5. It does not require the knowledge of any residual stress that may exist initially ( as a consequence of processing for instance).

In spite of all those attractive features, it should be reminded that the presented method does not give all the information regarding the high-cycle fatigue: neither the exact value of the endurance limit, nor the number of cycles to failure, are provided by the proposed approach (see [6] for recent progress in that direction). However, due to its simplicity, the proposed method could possibly be useful at least in the early stages of design.

\section{Appendix}

For the sake of completeness, we give in this Appendix a proof of Theorem 1. Consider a solution $\left(\varepsilon, \alpha, \sigma, A^{r}, A^{d}\right)$ to the evolution problem (1-10-11-13). By (11) we have

$$
A=A^{d}+A^{r}
$$

with

$$
\dot{\alpha} \in \partial I_{\mathscr{C}}\left(A^{d}\right) ; A^{r} \in \partial I_{\mathscr{T}}(\alpha)
$$

The positive quantity

Fig. 5 Map of $\left\|\varepsilon_{0}^{D}(x)\right\|$ on a stent cell (values are normalized with respect to the maximum value $\left\|\varepsilon_{0}^{D}\right\|_{\max }$ ).

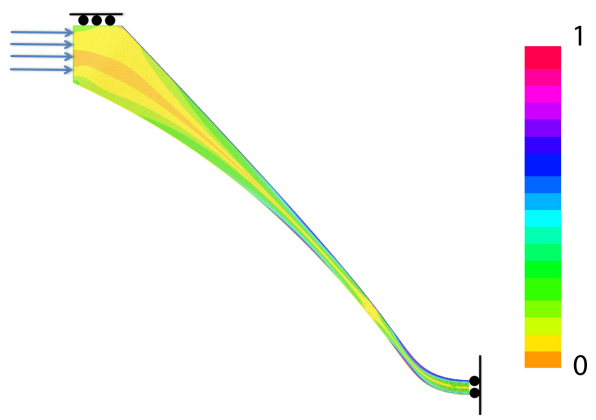




$$
D(t)=\int_{\Omega} A^{d} \cdot \dot{\alpha} d x
$$

can be interpreted as the rate of dissipated energy. Note that $D(t)$ is positive because of the principle of maximum dissipation (6) and the fact that the elasticity domain $\mathscr{C}$ contains the origin. Under the condition (16), we show in the following that the total dissipated energy $\int_{0}^{T} D(t) d t$ remains bounded as $T \rightarrow \infty$. To that purpose, consider the positive functional $W(t)$ defined as

$$
W(t)=\int_{\Omega} w\left(\varepsilon(t)-\varepsilon^{E}(t), \alpha(t)\right) d x
$$

By time-differentiation we have

$$
\dot{W}(t)=\int_{\Omega}\left[\left(\sigma-\sigma^{E}\right):\left(\dot{\varepsilon}-\dot{\varepsilon}^{E}-K: \dot{\alpha}\right)+f^{\prime}(\alpha): \dot{\alpha}\right] d x .
$$

Since $\operatorname{div}\left(\sigma-\sigma^{E}\right)=0$ in $\Omega,\left(\sigma-\sigma^{E}\right) . n=0$ on $\Gamma_{T}$ and $u-u^{E}=0$ on $\Gamma_{u}$, the principle of virtual power gives $\int_{\Omega}\left(\sigma-\sigma^{E}\right):\left(\dot{\varepsilon}-\dot{\varepsilon}^{E}\right) d x=0$. Therefore

$$
\dot{W}(t)=\int_{\Omega}\left[-K^{T}:\left(\sigma-\sigma^{E}\right)+f^{\prime}(\alpha)\right]: \dot{\alpha} d x
$$

which using (3) and (23) can be rewritten as

$$
\dot{W}(t)=-D(t)+\int_{\Omega}\left[-A^{r}+K^{T}: \sigma^{E}\right]: \dot{\alpha} d x .
$$

Let $\left(A_{*}^{r}, m\right)$ satisfying (16). Setting $A_{*}^{d}=m K^{T}: \sigma^{E}(t)-A_{*}^{r}$, we find

$$
\dot{W}(t)=-D(t)+\int_{\Omega}\left[-A^{r}+\frac{1}{m}\left(A_{*}^{d}+A_{*}^{r}\right)\right]: \dot{\alpha} d x .
$$

The property (16) shows that $A_{*}^{d} \in \mathscr{C}$ for $t>\tau$. Since $\dot{\alpha} \in \partial I_{\mathscr{C}}\left(A^{d}\right)$, the principle of maximum dissipation (6) gives

$$
\left(A^{d}-A_{*}^{d}\right): \dot{\alpha} \geq 0 .
$$

Moreover, since $A^{r} \in \partial I_{\mathscr{T}}(\alpha)$ and $\alpha \in \mathscr{T}$, Eq. (12) gives $A^{r}(t):\left(\alpha(t)-\alpha\left(t^{\prime}\right)\right) \geq 0$ for any $t^{\prime}$. In the limit $t^{\prime} \longrightarrow t$ with $t^{\prime}<t$, we obtain

$$
A^{r}: \dot{\alpha} \geq 0
$$

Combining (26-27) with (25) gives

$$
\dot{W}(t) \leq \frac{1-m}{m} D(t)+\frac{1}{m} \int_{\Omega} A_{*}^{r}: \dot{\alpha} d x .
$$

Since $A_{*}^{r}$ is time-independent, the time-integration of (28) on a time interval $[\tau, T]$ yields 


$$
(m-1) \int_{\tau}^{T} D(t) d t \leq m W(\tau)+\int_{\Omega} A_{*}^{r}:(\alpha(T)-\alpha(\tau)) d x
$$

where the property $W(T) \geq 0$ has been used. The set $\mathscr{T}$ being bounded, there exists a positive constant $K$ such that $\|\alpha\| \leq K$ for any $\alpha \in \mathscr{T}$. Therefore

$$
\int_{\Omega} A_{*}^{r}:(\alpha(t)-\alpha(\tau)) d x \leq 2 K \int_{\Omega}\left\|A_{*}^{r}\right\| d x
$$

Combining that inequality with (29) gives

$$
(m-1) \int_{\tau}^{T} D(t) d t \leq m W(\tau)+2 K \int_{\Omega}\left\|A_{*}^{r}\right\| d x
$$

The right-hand side of that inequality is independent of $T$. This proves that the dissipated energy $\int_{\tau}^{T} D(t)$ remains bounded as $T \longrightarrow+\infty$.

From there we can show (under some technical assumptions) that $\alpha(t)$ tends to a limit as $t \longrightarrow+\infty$. Assume that the elasticity domain $\mathscr{C}$ contains a ball of radius $r>0$ centered at the origin. In such a condition, we have $r \dot{\alpha}(t) /\|\dot{\alpha}(t)\| \in \mathscr{C}$ for any $t$. Using the principle of maximum dissipation (6), we find

$$
0 \leq \dot{\alpha}:\left(A^{d}-r \frac{\dot{\alpha}}{\|\dot{\alpha}\|}\right)
$$

Hence

$$
\|\dot{\alpha}\| \leq \frac{1}{r} A^{d}: \dot{\alpha}
$$

which after space integration gives

$$
\int_{\Omega}\|\dot{\alpha}\| d x \leq \frac{1}{r} D(t)
$$

Let $\mathbb{A}$ be the vectorial space in which $\alpha(x)$ takes values and let $L_{1}(\Omega, \mathbb{A})$ be the space of integrable functions with values in $\mathbb{A}$. The inequality (30) can be rewritten as

$$
\|\dot{\alpha}\|_{L_{1}(\Omega, \mathbb{A})} \leq \frac{1}{r} D(t)
$$

where $\|\cdot\|_{L_{1}(\Omega, \mathbb{A})}$ is the norm in $L_{1}(\Omega, \mathbb{A})$. Since $\int_{0}^{T} D(t)$ is bounded as $T \longrightarrow$ $+\infty$, the integral $\int_{0}^{T}\|\dot{\alpha}(t)\|_{L_{1}(\Omega, \mathbb{A})} d t$ converges as $T \longrightarrow+\infty$. From Riesz-Fischer theorem, the space $L_{1}(\Omega, \mathbb{A})$ is a Banach space. It follows (see [29] or Theorem 97 in [32]) that the integral $\int_{0}^{T} \dot{\alpha}(t) d t$ converges as $T \longrightarrow \infty$. Hence $\alpha(t)$ converges towards a limit as $T \longrightarrow \infty$. 
High-cycle fatigue in shape-memory alloys structures

\section{References}

1. Ahn YJ, Bertocchi E, Barber J (2008) Shakedown of coupled two-dimensional discrete frictional systems. J. Mech. Phys. Solids 56:3433-3440

2. Anand L, Gurtin M (2003) Thermal effects in the superelasticity of crystalline shpe-memory materials. J.Mech.Phys.Solids 51:1015-1058

3. Artioli E, Bisegna P (2015) An incremental energy minimization state update algorithm for $3 \mathrm{~d}$ phenomenological internal-variable sma constitutive models based on isotropic flow potentials. Int.J.Num.Meth.Eng. 105:197-220

4. Auricchio F, Petrini L (2004) A three-dimensional model describing stress-temperature induced solid phase transformations: solution algorithm and boundary value problems. Int.J.Num.Meth.Eng. 61:807-836

5. Auricchio F, Bonetti E, Scalet G, Ubertini F (2014) Theoretical and numerical modeling of shape memory alloys accounting for multiple phase transformations and martensite reorientation. Int.J.Plasticity 59: 30-54

6. Auricchio F, Constantinescu A, Menna C, Scalet G (2016) A shakedown analysis of high cycle fatigue of shape memory alloys. Int. J. Fatigue 87:112-123

7. Bhattacharya K (2003) Microstructure of martensite. Oxford University Press

8. Brézis H (1972) Opérateurs maximaux monotones et semigroupes de contractions dans les espaces de Hilbert.North-Holland, Amsterdam

9. Duda SH, Bosiers M, Lammer J, Scheinert D, Zeller T, Tielbeek A, Anderson J, Wiesinger B, Tepe G, Lansky A. (2005) Sirolimus-eluting versus bare nitinol stent for obstructive superficial femoral artery disease: the SIROCCO II trial. J. Vasc. Interv. Radiol. 16:331-338

10. Feng X, Sun Q (2007) Shakedown analysis of shape memory alloy structures. Int J. Plasticity 23:183-206

11. Frémond M (2002) Non-smooth thermomechanics. Springer

12. Govindjee S, Miehe C (2001) A multi-variant martensitic phase transformation model: formulation and numerical implementation. Comput. Meth. Appl. Mech. Engrg. 191: 215-238

13. Hackl K, Heinen R (2008) An upper bound to the free energy of $n$-variant polycrystalline shape memory alloys. J.Mech.Phys.Solids 56:2832-2843

14. Hecht F (2012) New development in FreeFem++. J. Numer. Math. 20:251-265

15. Koiter WT (1960) General theorems for elastic-plastic solids. In: Progress in solid mechanics

16. Mandel J, Zarka J, Halphen B (1977) Adaptation d'une structure elastoplastique a ecrouissage cinematique. Mech.Res. Commun. 4:309-314

17. Manson SS, Halford GR (2006) Fatigue and durability of structural materials. ASM International

18. Melan E (1936) Theorie statisch unbestimmter Systeme aus ideal-plastischen Baustoff. Sitz.Berl.Ak.Wiss. 145: 195-218

19. Peigney M (2008) Recoverable strains in composite shape memory alloys, J. Mech. Phys. Solids 56:360-375

20. Peigney M (2009) A non-convex lower bound on the effective free energy of polycrystalline shape memory alloys. J.Mech.Phys.Solids 57: 970-986

21. Peigney M (2010) Shakedown theorems and asymptotic behaviour of solids in non-smooth mechanics. Eur. J. Mech. A 29:784-793

22. Peigney M, Seguin JP, Hervé-Luanco E (2011) Numerical simulation of shape memory alloys structures using interior-point methods.Int. J. Sol. Struct. 48:2791-2799

23. Peigney M, Seguin JP (2013) An incremental variational approach to coupled thermomechanical problems in anelastic solids. application to shape-memory alloys. Int. J. Sol. Struct.50:4043-4054

24. Peigney M (2013) On the energy-minimizing strains in martensitic microstructures-Part 1: Geometrically nonlinear theory. J.Mech.Phys.Solids 61:1489-1510

25. Peigney M (2013) On the energy-minimizing strains in martensitic microstructures-Part 2: Geometrically linear theory. J.Mech.Phys.Solids 61:1511-1530 
26. Peigney M (2014) On shakedown of shape memory alloys structures, Ann. Solid Struct. Mech. On shakedown of shape memory alloys structures 6:17-28

27. Peigney M (2014) Shakedown of elastic-perfectly plastic materials with temperaturedependent elastic moduli, J. Mech. Phys. Solids 71:112-131

28. Pelton AR, Schroeder V, Mitchell MR, Gong XY, Barney M, Robertson SW (2008) Fatigue and durability of Nitinol stents . J.Mech.Behaviour.Bio.Mat. 1:153-164

29. Rudin W (1987) Real and Complex Analysis.McGraw-Hill, Boston

30. Sabet S, Mlekusch W, Amighi J, Minar E, Schillinger M (2005) Primary patency of longsegment self-expanding nitinol stents in the femoropopliteal arteries. J. Endovasc. Ther. 12:612

31. Scalet G, Peigney M (2017) A robust and efficient radial return algorithm based on incremental energy minimization for the 3D Souza-Auricchio model for shape memory alloys. Eur. J. Mech. A 61:364-382

32. Schwartz L (1967) Analyse mathmatique, vol. 94.Hermann, Paris

33. Souza A, Mamiya E, Zouain N (1998) Three-dimensional model for solids undergoing stressinduced phase transformations. Eur.J.Mech. A 17:789-806

34. Symonds PS (1951) Shakedown in continuous media. J. Appl. Mech. 18:85-89 